\title{
Treatment of chemoresistant cell lines with indolinone-based small molecules
}

\author{
Aleksandra Sagaidak ${ }^{1}$, Milena Lvova $^{1}$, Aleksander Garabadzhiu ${ }^{1}$, and Angelina \\ Romanova, ${ }^{1, *}$ \\ ${ }^{1}$ St. Petersburg state institute of technology (technical univercity), Laboratory of molecular \\ pharmacology, Moskovskii pr, 26, St. Petersburg, Russia
}

\begin{abstract}
Reduced delivery of the drug into the cell due to increased activity of specific transporter P-glycoprotein is one of the main mechanisms of drug resistance development. The inhibition of the activity of such a pump increases the intracellular concentration of the drug and contributes to cancer cell death. The combination of factors that allows one to overcome genetically determined resistance and to trigger apoptosis in one small molecule compound can lead to the development of new type of drugs for personalized therapy of chemoresistant tumors. In the course of work on optimization of MDM2 inhibitors based on indolinones and isoindolinones, we found fragments of the structure that can be modified with minimal risk of a decrease in the target activity. The combination of in silico and in vitro methods allowed the selection of compounds that showed strong binding to the target sites of P-glycoprotein and MDM2, and a good combination of solubility - membrane-active properties, which implies high bioavailability of the drug.
\end{abstract}

\section{Introduction}

Multiple drug resistance (MDR) of cancer cells, when the tumor is insusceptible to a wide range of drugs with a different mechanism of action, is an important issue in the chemotherapy of proliferative diseases. There are primary drug resistance, that is initially inherent to a tumor, and secondary drug resistance. Cell phenotypes with secondary resistance manifest in the case of tumors that are initially sensitive to the therapy. At the same time, proliferation of cancer cells that survived after the first line of the therapy leads to the formation of a secondary tumor, which may be insensitive to the primary treatment regimen. This is manifested as the progression of the tumor at the next line of the therapy after complete or partial regression or stabilization. Thus, chemotherapy, which was successful at the beginning of the treatment, becomes ineffective. The frequency of relapses due to drug resistance ranges from 7 to $75 \%$ and varies depending on the nature and location of the tumor. In the case of ovarian cancer, about $80 \%$ of tumors are initially susceptible to chemotherapy, but in $75 \%$ of cases secondary resistance develops under used chemotherapy, at the same time the tumor often becomes less sensitive both to radiotherapy and platinum drugs.

\footnotetext{
*Corresponding author: agurangelina@mail.ru
} 


\section{Metarials and methods}

In recent decades, due to the achievements in pharmacology there is a steady broadening of the spectrum of proliferative diseases that respond to chemotherapy. In this regard, the prevention of secondary drug resistance and overcoming chemoresistance of primary tumors is an important issue of modern oncology, which can be solved by developing new drugs for personalized therapy.

Tumor chemoresistance is determined by a number of causes, among them the following are noted [1]:

- significant change in the ratio of pro- and anti-apoptotic proteins;

- accelerated deactivation of the drug by various cellular enzymes;

- disruption of drug delivery to the cell.

Reduced delivery of the drug into the cell due to increased activity of specific transport proteins is recognized as one of the main mechanisms of drug resistance development [2]. P-glycoprotein (Pgp) is the most important transport protein. Although Pgp is expressed in cells whose function is related to detoxification and elimination of xenobiotics, in particular, in intestinal epithelium, liver, immunocompetent cells, excessive activity of the efflux mechanism leads to the formation of multidrug resistance of cancer cells [3]. So, the inhibition of the activity of such a pump increases the intracellular concentration of the drug and contributes to cancer cell death.

The identification of small molecule Pgp inhibitors among natural compounds [3] and the development of their analogues [4] are the most used approaches to the search for drugs that contribute to the suppression of tumor chemoresistance. Such substances have no independent therapeutic value and are used only in combination with anticancer drugs. Since Pgp inhibitors do not exhibit their own therapeutic properties, the development of a molecule capable of simultaneously inhibiting Pgp and proliferation of cancer cells is relevant.

Previously, compounds combining the structures of Pgp inhibitors and anticancer agents have been proposed (thioxanthones [5], furoxanes [6]). It should be noted that the structure of the described drugs consists of two fragments, showing different types of activity, connected by a linker. Although such an approach to the development of multitarget drugs seems formally obvious, it usually leads to very modest results. In turn, we implement an integrated approach using modern methods of medical chemistry, including computer modeling, synthesis, in vitro and in vivo studies of the biological activity with parallel optimization of lead compounds. The basis for the developed small molecule structures is not the combination of dissimilar substrates, but the rational design of a multitarget compound capable of simultaneous inhibiting Pgp and the target proteinprotein interaction due to the targeted development. This approach allows us to develop an agent that is effective against both chemosensitive and chemoresistant tumors, and to conduct monotherapy of tumors in patients with multidrug resistance.

The use of drugs with different mechanisms of action is most effective in the case of pathologies of a complex nature, such as cancer, various neurodegenerative diseases, inflammatory processes, mental diseases [7,8]. Current therapeutic regimens suggest the combined use of 2 or more drugs in the case of complex diseases; however, multitarget therapy is preferable because it has more predictable pharmacokinetics and tolerance, significantly reduced risks of side effects. For the development of a multitarget anticancer drug we propose to use the inhibition of the MDM2-p53 protein-protein interaction [9-13], which has been actively studied in the last decade, as a target together with the Pgp inhibition.

The p53 signaling protein plays an important role in determining the fate of individual cells of a multicellular organism. It triggers the process of apoptosis (programmed cell 
death) when the genetic information of the cell is damaged. Proliferative diseases are caused by a decrease in the expression of the p53 protein, caused in most cases by the hyperactivity of the MDM2 protein, which directly binds to p53 and does not allow it to manifest its oncosuppressive properties. Reactivation of $\mathrm{p} 53$ by inhibiting MDM2 is one of the most relevant modern strategies for targeted oncotherapy. The combination of factors to overcome genetically determined resistance and induction of apoptosis in one lowmolecular compound can lead to the creation of new type of drugs for personalized therapy of chemoresistant tumors. Proliferative diseases are determined by a decrease in expression of the p53 protein caused in most cases by the hyperactivity of the MDM2 protein, which directly binds to p53 and does not allow it to manifest its tumor suppressor properties. Reactivation of $\mathrm{p} 53$ by inhibiting MDM2 is one of the most relevant modern strategies for targeted oncotherapy.

The combination of factors that allows one to overcome genetically determined resistance and to trigger apoptosis in one small molecule compound can lead to the development of new type of drugs for personalized therapy of chemoresistant tumors. In particular, good results can be expected in the case of the treatment of solid tumors: sarcomas and blastomas of different localization, since both tariquidar, the Pgp inhibitor, and MDM2 inhibitors undergo clinical trials in this direction. The mentioned inhibitors are tested in the combination with doxorubicin. In the first case, the target effect is the absence of efflux of the genotoxic agent and, as a consequence, a decrease in the probability of the resistance development. In the second case, the main aim is to preserve increased expression of the proapoptotic p53 protein. Thus, the combined use of new multitarget agents with well-studied chemotherapy agents is very promising.

The Pgp activity determines tumor cell resistance to many anticancer drugs, in particular, anthracycline antibiotics, including doxorubicin, and plant alkaloids, such as drugs of the Taxol group, as well as to many other substances: fluorescent dyes, puromycin, gramicidin D, and others $[14,15]$. This extremely low specificity is explained by the features of the Pgp protein function, which is ensured by the presence of several binding sites for substances of various structures.

The first step in designing a multitarget drug was the computer simulation of the interaction of small molecule compounds with MDM2 and Pgp using the software package from Schrödinger. We analyzed the protein models presented in the Protein Data Bank and identified the Pgp affinity region for potential inhibitors. An optimized model was subsequently used for in silico assessment of the affinity of candidate compounds for Pgp. In the case of MDM2, for the virtual stage of work, we used models that we tested earlier in the development of MDM2 inhibitors [11, 12, 16, 17].

The library was based on classes of compounds studied in our laboratory, which in in vitro experiments showed activity comparable to the activity of substances at the stage of clinical trials [18, 19] (Fig. 1).
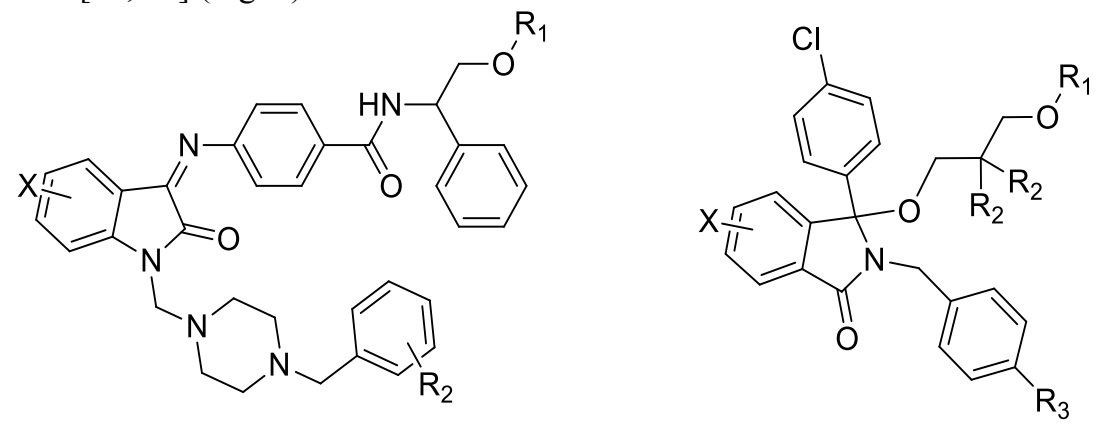

Fig. 1. Promising indolinone and isoindolinone representatives. 
In the course of work on optimization of MDM2 inhibitors based on indolinones and isoindolinones [19-21], we found fragments of the structure that can be modified with minimal risk of a decrease in the target activity, i.e. ability to inhibit the formation of the complex MDM2-p53. In particular, the esterification of the -R1 hydroxyl group (Fig. 1) does not disrupt key interactions with the MDM2 protein cavity, which allows modification of the molecule to increase its affinity for the Pgp target site without decreasing the affinity for MDM2. On the other hand, the variation in the binding of the developed compounds to the MDM2 cavity makes it possible to select -X substituents in the indole core of the compounds (Fig. 1).

The combination of in silico and in vitro methods allowed the selection of two dozen of the five thousand compounds that make up the focused base for subsequent synthesis. These compounds showed, on the one hand, strong binding to the target sites of Pgp and MDM2 (high GoldScore function values with adequate stacking methods), and, on the other hand, a good combination of solubility - membrane-active properties, which implies high bioavailability of the drug.

\section{Results}

We showed that indolinone derivatives can effectively induce apoptosis of cancer cells by the example of U2OS (human osteosarcoma) and HEK293T (human embryonic kidney) cell lines with different p53 status, at the same time the mechanism of the drugs was confirmed using recombinant protein models of p53 and MDM2 [9, 12, 18]. On the other hand, substances based on the indolinone scaffold confirmed their effectiveness in preventing and/or slowing down the development of multidrug resistance; promising results were obtained not only in experiments in cells, but also in xenografts [22, 23].

The data obtained indicate high efficiency of potential multitarget anticancer drugs that are able to:

a) provoke cancer cell apoptosis by inhibiting MDM2 and reactivating p53;

b) inhibit Pgp and, accordingly, increase the sensitivity of cancer cells to drugs that are Pgp substrates, such as doxorubicin.

The combination of the discussed properties in one molecule will allow one to develop a drug for personalized therapy of tumors with primary and secondary chemoresistance caused by the activity of both Pgp and MDM2. The introduction of such a drug into the practice will significantly expand the range of oncological diseases amenable to the therapy, and will simplify the treatment regimen by reducing the number of used drugs administered simultaneously up to the monotherapy.

This work was financially supported by the Russian Science Foundation (project no. 16-13-10358).

\section{References}

1. G. Housman, S. Byler, S. Heerboth, et al. Drug resistance in cancer: an overview, Cancers 6, 3, 1769-1792 (2014)

2. J.P. Gillet, M.M. Gottesman, Overcoming multidrug resistance in cancer: 35 years after the discovery of $A B C B 1$, Drug resistance updates: reviews and commentaries in antimicrobial and anticancer chemotherapy 15, 1-2, 2-4 (2012)

3. A.I. Svirnovsky, S.A. Grigorovich, Pleiotropic resistance of tumor cells to therapeutic effects in B-cell lymphoproliferative diseases, Medical news 9, 5-16 (2005) 
4. S.C. Nicklisch, S.D. Rees, A.P. McGrath, et al. Global marine pollutants inhibit Pglycoprotein: Environmental levels, inhibitory effects, and cocrystal structure, Science advances 2(4), e1600001 (2016)

5. A. Palmeira, M.H. Vasconcelos, A. Paiva, et al. Dual inhibitors of P-glycoprotein and tumor cell growth: (re)discovering thioxanthones, Biochemical pharmacology 83, 1, 57-68 (2012)

6. N.A. Colabufo, M. Contino, F. Berardi, et al. A new generation of MDR modulating agents with dual activity: $P$-gp inhibitor and iNOS inducer agents, Toxicology in vitro 25, 1, 222-230 (2011)

7. A. Talevi, Multi-target pharmacology: possibilities and limitations of the «skeleton key approach» from a medicinal chemist perspective, Front Pharmacol. 6, 205, 1-7 (2015)

8. D.S. Novikova, A.V. Garabadzhiu, G. Melino, N.A. Barlev, V.G. Tribulovich, Smallmolecule activators of AMP-activated protein kinase as modulators of energy metabolism, Russ. Chem. Bull. 64, 7, 1497-1517 (2015)

9. T.A. Grigoreva, D.S. Novikova, A.V. Petukhov, M.A. Gureev, A.V. Garabadzhiu, G. Melino, N.A. Barlev, V.G. Tribulovich, Proapoptotic modification of substituted isoindolinones as MDM2-p53 inhibitors, Bioorg. Med. Chem. Lett. 27, 5197-5202 (2017)

10. T.A. Grigoreva, A.V. Garabadzhiu, V.G. Tribulovich, Diastereotopic derivatives of chiral alkoxyisoindolinones, Russ. J. Gen. Chem. 86, 11, 2454-2461 (2016)

11. D.S. Novikova, T.A. Grigoreva, G.S. Ivanov, G. Melino, N.A. Barlev, V.G. Tribulovich, Activating effect of 3-benzylidene oxindoles on AMPK: from computer simulation to high-content screening, Chem. Med. Chem. 15 (2020)

12. O. Fedorova, A. Daks, V. Petrova, A. Petukhov, L. Lezina, O. Shuvalov, P. Davidovich, D. Kriger, E. Lomert, D. Tentler, V. Kartsev, B. Uyanik, V. Tribulovich, O. Demidov, G. Melino, N.A. Barlev, Novel isatin-derived molecules activate p53 via interference with Mdm2 to promote apoptosis, Cell Cycle 17, 15, 1917-1930 (2018)

13. M. Krasavin, M.A. Gureyev, D. Dar'in, O. Bakulina, M. Chizhova, A. Lepikhina, D. Novikova, T. Grigoreva, G. Ivanov, A. Zhumagalieva, A.V. Garabadzhiu, V.G. Tribulovich, Design, in silico prioritization and biological profiling of apoptosisinducing lactams amenable by the Castagnoli-Cushman reaction, Bioorg. Med. Chem. 26, 9, 2651-2673 (2018)

14. D.J. Booser, G.N. Hortobagyi, Anthracycline antibiotics in cancer therapy. Focus on drug resistance, Drugs 47, 2, 223-258 (1994)

15. S.M. He, R. Li, J.R. Kanwar, S.F. Zhou, Structural and functional properties of human multidrug resistance protein 1 (MRPI/ABCC1), Current medicinal chemistry 18, 3, 439-481 (2011)

16. T. Grigoreva, A. Romanova, A. Sagaidak, S. Vorona, D. Novikova, V. Tribulovich, Mdm2 inhibitors as a platform for the design of P-glycoprotein inhibitors, Bioorganic \& Medicinal Chemistry Letters 30, 18, 127424 (2020)

17. M. Gureev, D. Novikova, T. Grigoreva, S. Vorona, A. Garabadzhiu, V. Tribulovich, Simulation of MDM2 N-terminal domain conformational lability in the presence of imidazoline based inhibitors of MDM2-p53 protein-protein interaction, J. Comput. Aided. Mol. Des. 34, 1, 55-70 (2020)

18. T.A. Grigoreva, D.S. Novikova, M.A. Gureev, A.V. Garabadzhiu, V.G. Tribulovich, Amino acids as chiral derivatizing agents for antiproliferative substituted $N$-benzyl isoindolinones, Chirality 30, 6, 785-797 (2018) 
19. D.S. Novikova, T.A. Grigoreva, A.A. Zolotarev, A.V. Garabadzhiu, V.G. Tribulovich, Advanced palladium free approach to the synthesis of substituted alkene oxindoles: Via aluminum-promoted Knoevenagel reaction, RSC Advances 8, 60, 34543-34551 (2018)

20. D.D. Orlova, D.S. Novikova, A.V. Garabadzhiu, V.G. Tribulovich, A study on hydrolytic stability of isatin N-Mannich bases, Russ. J. Gen. Chem. 88, 1, 48-56 (2018)

21. P. Davidovich, D. Novikova, V. Tribulovich, S. Smirnov, V. Gurzhiy, G. Melino, A. Garabadzhiu, First X-ray structural characterization of isatin Schiff base derivative. NMR and theoretical conformational studies, J. Mol. Struct. 1075, 450-455 (2014)

22. X. Yang, J. Shen, Y. Gao, et al. Nsc23925 prevents the development of paclitaxel resistance by inhibiting the introduction of Pglycoprotein and enhancing apoptosis, Int. J. Cancer 137, 8, 2029-2039 (2015)

23. J. Wang, N. Seebacher, H. Shi, Q. Kan, Z. Duan, Novel strategies to prevent the development of multidrug resistance (MDR) in cancer, Oncotarget 8, 48, 84559-84571 (2017) 